\section{Fetal Diagnosis \\ and Therapy}

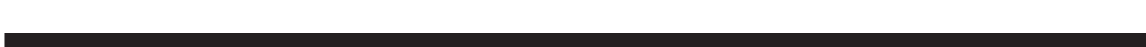

The editors of Fetal Diagnosis and Therapy gratefully acknowledge the assistance of the following referees from August 1, 2008 to November 30, 2009

N. Scott Adzick, Philadelphia, Pa. Judith Alder, Basel Lindsey Allan, London Janyne Althaus, Baltimore, Md. Eugenia Antolin, Madrid Birgit Arabin, Zwolle Norbert Arnold, Kiel Robert H. Ball, Salt Lake City, Utah Christian Bamberg, Berlin José Luis Bartha Rasero, Cádiz Seher Basaran, Capa, Istanbul Ahmet Alexander Baschat, Baltimore, Md. Haim Bassan, Tel Aviv Beryl Benacerraf, Boston, Mass. Alexandra Benachi, Paris Kurt Benirschke, La Jolla, Calif. Brigitte Benzacken, Bondy Harm-Gerd Blaas, Trondheim Karin J. Blakemore, Baltimore, Md. Eran Bornstein, New York, N.Y. Ioana Bratu, Alberta Luc Breysem, Leuven Mary Brindle, Calgary Richard N. Brown, Montreal Christoph A. Bührer, Berlin The-Hung Bui, Stockholm Luis Cabero-Roura, Barcelona Geraldine Canny, Lausanne Lionel Carbillon, Bondy Frank Chen, Berlin Yoshihide Chiba, Osaka Ramen H. Chmait, Los Angeles, Calif. Michael Christiansen, Copenhagan Tere Cobo, Barcelona David E. Cohen, Philadelphia, Pa. Erich Cosmi, Padua Fatima Crispi, Barcelona Enrico Danzer, Philadelphia, Pa. Jan A. Deprest, Leuven Roland Devlieger, Leuven Gianna Di Cienzo Hüsler, Basel Gian Carlo Di Renzo, Perugia Marc Dommergues, Paris Elisa Doné, Leuven Mariusz Dubiel, Bydgoszcz Joachim W. Dudenhausen, Berlin Stephen P. Emery, Pittsburgh, Pa.
Mark I. Evans, New York, N.Y. Francesc Figueras, Barcelona Isabel Filges, Basel

Nicholas M. Fisk, Brisbane, Qld. Alan W. Flake, Philadelphia, Pa. W.D. Andrew Ford, Adelaide Jean-Pierre Fryns, Leuven Alain Gagnon, Vancouver Alberto Galindo, Madrid Denis Gallot, Clermont-Ferrand Helena M. Gardiner, London Verena Geissbühler, Basel Fabio Ghezzi, Varese Tullio Ghi, Bologna Norman A. Ginsberg, Chicago, Ill. Olga Gómez, Barcelona Anna Goncé, Barcelona Eduard Gratacós, Barcelona Andrea L. Greiner (Fick), Iowa City, Iowa Felix Häberlin, St. Gallen Narelle Hadlow, Perth

Michael R. Harrison, San Francisco, Calif. Moritz Hartog, Basel

Ivo Markus Heer, Kiel Wolfgang Henrich, Berlin Edgar Hernández-Andrade, Barcelona William Allan Hogge, Pittsburgh, Pa. Wolfgang Holzgreve, Freiburg Irene Hösli, Basel

Evelyn Huhn, Basel Berthold Huppertz, Graz Jon A. Hyett, Sydney Lurie W. Iosif, Baltimore, Md. Russell W. Jennings, Boston, Mass. Mark Paul Johnson, Philadelphia, Pa. Ann M. Johnson, Philadelphia, Pa. Connie Jorgensen, Copenhagen

Jean-Marie Jouannic, Paris

Franz Kainer, München

Masato Katsuyama, Kyoto

Peter Kaufmann, Aachen

Ingo Kennerknecht, Münster

Peter C.W. Kim, Toronto

André B. Kind, Bern

Pertti Kirkinen, Tampere

David A. Krantz, Melville, N.Y.

Marcus Krüger, Freiburg
Asim Kurjak, Zagreb

Juozas Kurmanavicius, Zürich

Jean-Martin Laberge, Montreal

Sylvie Langlois, Vancouver

Olav Lapaire, Basel

Tze Kin Lau, Shatin, Hong Kong

Hanmin Lee, San Francisco, Calif.

Tobias J. Legler, Göttingen

Shlomo Lipitz, Tel-Hashomer

Gerald S. Lipshutz, Los Angeles, Calif.

Anna Locatelli, Milano

Enrico Lopriore, Leiden

Teresa Loureiro, S. Mamede de Infesta

Gwendolin Manegold, Basel

Giancarlo Mari, Detroit, Mich.

Joseph-Maria Martinez, Madrid

Teresa Martino, Baltimore, Md.

Holger Maul, Heidelberg

Lynne G. Maxwell, Philadelphia, Pa.

Ron Maymon, Zerifin

Johannes Mayr, Basel

Fionnuala M. McAuliffe, Dublin

Erik C. Michelfelder, Cincinnati, Ohio

Peter Miny, Basel

Hiten Mistry, London

Francisca Molina Garcia Molina Garcia, Granada

Giovanni Monni, Cagliari

Thomas R. Moore, San Diego, Calif.

Rachel Katherine Morris, Birmingham

Jonathan Morris, Sydney

Anne-Claude Müller Brochut, Bern

Allan Nadel, Boston, Mass.

Anwar Nassar, New York, N.Y.

H. Norman Noe, Memphis, Tenn.

Anthony Odibo, St. Louis, Mo.

Dick Oepkes, Leiden

Caroline Mackie Ogilvie, London

Lucas Otaño, Buenos Aires

Dario Paladini, Napoli

Ricardo S. Palma-Dias, Carlton

Aris T. Papageorghiou, London

Zoltán Papp, Budapest

Alberto Peña, Cincinnati, Ohio

Greg B. Peters, Westmead, Sydney

Michael B. Petersen, Piraeus

Walter Plasencia, Canary Islands 
Lawrence D. Platt, Los Angeles, Calif.

Ritsuko K. Pooh, Osaka

Bienvenido Puerto, Barcelona

Luigi Raio, Bern

Helga Rehder, Wien

Robert Resnik, San Diego, Calif.

David L. Rimoin, Los Angeles, Calif.

Giuseppe Rizzo, Roma

Roberto J. Romero, Detroit, Mich.

Rodrigo Ruano, São Paulo

Magdalena Sanz, Barcelona

Elena Scazzocchio Dueñas, Barcelona

Dörte Schäfer, Basel

Matthias Scheier, Innsbruck

Dominique Van Schoubroeck, Leuven

Akihiko Sekizawa, Tokyo

Waldo Sepulveda, Santiago

Norman H. Silverman, Palo Alto, Calif.

Joe Leigh Simpson, Miami, Fla.

Lynn L. Simpson, New York, N.Y.

Erik D. Skarsgard, Vancouver

Maja Slakovic, Sarajevo
Rosalinde Snijders, Rotterdam

Jiri Sonek, Dayton, Ohio

Sarah T. South, Salt Lake City, Utah

Catherine Y. Spong, Bethesda, Md.

Werner Stein, Göttingen

Joanne Lynne Stone, New York, N.Y.

Erin Story Oehler, Baltimore, Md.

Alexander Strauss, Kiel

Daniel V. Surbek, Bern

Mitsuyo Tanemura, Nagoya

Baskaran (Basky) Thilaganathan, London

Ilan E. Timor-Tritsch, New York, N.Y.

Monya Todesco-Bernasconi, Aarau

Ants Toi, Toronto

Brian J. Trudinger, Westmead

Dimitrios A. Tsakiris, Basel

Nancy B.Y. Tsui, Hong Kong

Kiyomi Tsukimori, Fukuoka

Boris A. Tutschek, Düsseldorf

Jun Udagawa, Izumo

Inge L. van Kamp, Leiden
John M.G. van Vugt, Amsterdam

Tim Vanmieghem, Leuven

Voula Velissariou, Athens

Klara A. Vergesslich-Rothschild, Wien

Eva J. Visca, Basel

Ida Vogel, Aarhus

Cora Alexandra Vökt, Basel

Constantin S. von Kaisenberg, Kiel

Ursula von Mandach, Zürich

Ronald J. Wapner, New York, N.Y.

Steven L. Warsof, Virginia Beach, Va.

Jonathan Waters, London

Ling Y. Wee, London

Magnus Westgren, Stockholm

Paul Williams, Sydney

R. Douglas Wilson, Calgary

Juriy W. Wladimiroff, Rotterdam

Elizabeth Wood Denne, Baltimore, Md.

Jie Xu, Ontario

Shi-Joon Yoo, Toronto

Yefei Zhu, Nanjing 